\title{
Seminal vesicles and diabetic neuropathy: ultrasound evaluation in patients with couple infertility and different levels of glycaemic control
}

\author{
Sandro La Vignera, Rosita A Condorelli, Enzo Vicari, Rosario D’Agata and Aldo E Calogero
}

The aim of this study was to evaluate the ultrasound characteristics of the seminal vesicles (SVs) of infertile patients with diabetes mellitus (DM) and diabetic neuropathy (DN) and to investigate possible changes in ultrasound characteristics related to glycaemic control. To accomplish this, 45 infertile patients with type $2 \mathrm{DM}$ and symptomatic DN were selected. Twenty healthy fertile men and 20 patients with idiopathic oligoasthenoteratozoospermia without DM represented the control groups. DM patients were arbitrarily divided into three groups according to glycaemic control level $(A=$ glycosylated haemoglobin $<7 \%$; $B=$ glycosylated haemoglobin between $7 \%$ and $10 \% ; C=$ glycosylated haemoglobin $>10 \%$ ). Patients underwent prostate-vesicular transrectal ultrasonography and sperm analysis. The following SV ultrasound parameters were recorded: (i) body antero-posterior diameter (APD); (ii) fundus APD; (iii) parietal thicknesses of the right and left SVs; and (iv) the number of polycyclic areas within both SVs. We then calculated the following parameters: (i) fundus/body (F/B) ratio; (ii) difference of the parietal thickness between the right and the left SV; and (iii) pre- and post-ejaculatory APD difference. All DM patients had a higher F/B ratio compared to controls $(P<0.05)$. Group $C$ had a higher $F / B$ ratio compared to the other DM groups $(P<0.05)$. All DM patients had a lower pre- and post-ejaculatory difference of the body SV APD compared to controls $(\boldsymbol{P}<0.05)$. Groups A and B had a similar pre- and post-ejaculatory difference of the body SV APD, whereas this difference was lower in Group C $(\boldsymbol{P}<0.05)$. In conclusion, infertile DM patients with DN showed peculiar SV ultrasound features suggestive of functional atony, and low glycaemic control was associated with greater expression of these features. Asian Journal of Andrology (2011) 13, 872-876; doi:10.1038/aja.2011.47; published online 1 August 2011

Keywords: diabetes; glycaemic control; infertility; neuropathy; seminal vesicles

\section{INTRODUCTION}

We recently evaluated the ultrasound characteristics of the seminal vesicles (SVs) in patients with diabetes mellitus (DM) by transrectal prostate-vesicular ultrasonography before and $1 \mathrm{~h}$ after ejaculation following 1 day of sexual abstinence. In a previous study, ${ }^{1}$ infertile patients with type $2 \mathrm{DM}$ and no other known cause of sperm parameter abnormalities were selected and evaluated, and the results were compared with those of healthy patients with idiopathic infertility or infertile patients with male accessory gland infections (MAGI), a wellstudied clinical model of seminal vesicle inflammation. The following SV ultrasound parameters were evaluated: (i) body antero-posterior diameter (APD); (ii) fundus APD; (iii) parietal thickness of both seminal vesicles; (iv) number of polycyclic areas within both seminal vesicles; (v) fundus/body (F/B) ratio; (vi) difference in parietal thickness between the two seminal vesicles; and (vii) pre- and postejaculatory APD difference. Diabetic patients had a higher F/B ratio compared with controls or patients with MAGI. Patients with MAGI were the only group showing an elevated number of polycyclic areas. Controls and patients with MAGI had a similar pre- and postejaculatory difference of the body SV APD, whereas this difference was less pronounced in patients with DM. In summary, this study demonstrated that infertile patients with DM have peculiar SV ultrasound features which differ from those seen in patients with vesiculitis. In a subsequent study, ${ }^{2}$ we evaluated the SV ultrasound features described above in patients with DM and diabetic neuropathy (DN) and their possible changes in relation to the duration of DM. Infertile patients with type $2 \mathrm{DM}$ and symptomatic $\mathrm{DN}$ were divided into three groups according to disease duration: group A: $<5$ years; Group B: $5-$ 15 years; Group C: $>15$ years. All diabetic patients had a higher F/B ratio and a lower pre- and post-ejaculatory difference of the body SV APD compared with controls. Patients with the longest disease duration (Group C) showed a higher F/B ratio and a lower pre- and postejaculatory difference of the body SV APD compared with the other diabetic groups. The latter parameter was similar in patients of the Groups A and B. These findings suggested that infertile diabetic patients with neuropathy have SV ultrasound features suggestive of functional atony, and the duration of the disease is associated with worse ultrasound findings. The unchanged SV APD after ejaculation in patients with DM is similar to our observations in patients with alcoholic or dysvitaminosis neuropathy. ${ }^{3}$ Subsequently, we reported 
that this SV functional atony is associated with a higher leukocyte concentration in the seminal fluid. Therefore, SV functional atony may favour the onset of a chronic inflammatory response of the prostate-ampullo-vesicular tract in these young infertile patients. ${ }^{3}$ In the present study, we further investigated these findings by evaluating whether the glycaemic control may influence the characteristics of the ultrasound findings.

\section{MATERIALS AND METHODS}

A total of 45 infertile patients with DM and DN were selected for this study and divided into three groups:

- Group A comprised 15 diabetic patients with serum levels of glycosylated haemoglobin $<7 \%$. The mean age of this group was $44.0 \pm 3.0$ years (range: $36-48$ years).

- Group B was composed of 15 diabetic patients with serum levels of glycosylated haemoglobin between $7 \%$ and $10 \%$. The mean age of Group B was $42.0 \pm 2.5$ years (range: $35-46$ years).

- Group C included 15 diabetic patients with serum levels of glycosylated haemoglobin $>10 \%$. These patients had a mean age of $41.0 \pm 2.0$ years (range: $32-48$ years).

Diabetic patients had a mean body mass index (BMI) of $27 \pm 2 \mathrm{~kg} \mathrm{~m}^{-2}$ (range: $25-30 \mathrm{~kg} \mathrm{~m}^{-2}$ ). All patients examined had symptomatic neuropathy, stage 2 according to the Dyck's classification. ${ }^{4,5}$ Stage 2 of this four-stage classification is diagnosed when at least two of the following abnormalities are present: moderate alterations detected at the physical exam, and/or electrophysiological, cardiovascular and/or autonomic tests.

The following two groups of patients were retrospectively selected and included in the study as diseased and true controls:

- Patients with oligoasthenoteratozoospermia (OAT): 20 patients with idiopathic OAT and without DM. They had a mean age of $42.0 \pm 0.4$ years (range: $34-47$ years) and a mean BMI of $27.0 \pm 0.5 \mathrm{~kg} \mathrm{~m}^{-2}$ (range: $22-30 \mathrm{~kg} \mathrm{~m}^{-2}$ );

- Fertile control group data analysis: 20 healthy fertile men. They had a mean age of $40.0 \pm 1.2$ years (range: $30-50$ years) and a mean BMI of $28.0 \pm 1.5 \mathrm{~kg} \mathrm{~m}^{-2}$ (range: $24-30 \mathrm{~kg} \mathrm{~m}^{-2}$ ).

Each patient enrolled in the study underwent to an accurate andrological diagnostic work-up including physical examination, sperm analysis $\left(\mathrm{WHO}, 1999^{6}\right)$ and ultrasound scans. Healthy fertile men who underwent transrectal ultrasound evaluation were selected from patients coming to our centre for chronic pelvic pain without diagnostic evidence of MAGI.
Exclusion criteria are as follows: (i) ultrasound signs of proximal (epididymal) and/or distal (ampullo-prostato-vesicular) obstruction; (ii) ultrasound and/or microbiological signs of male accessory gland infections; (iii) hormonal disorders including hypogonadism (total testosterone $<3 \mathrm{ng} \mathrm{ml}^{-1}$ or $10.4 \mathrm{nmoll}^{-1}$ ), ${ }^{7}$ hyperprolactinemia (prolactin $\left.>25 \mathrm{ng} \mathrm{ml}^{-1}\right)^{8}$ and hypothyroidism $\left(\mathrm{TSH}>4.5 \mu \mathrm{U} \mathrm{ml}^{-1}\right.$ ). ${ }^{9}$ Endocrine factors can affect ejaculatory time and function, ${ }^{10}$ and we therefore arbitrarily chose to exclude these patients to minimize factors promoting stasis in the seminal vesicles. Table 1 shows the serum hormone concentrations of these patients.

In addition, we excluded subjects from the study meeting any of the following criteria: cigarette smoking, alcohol consumption, occupational chemical exposure, fever or drug use within 3 months prior to enrolment in this study, azoospermia, testicular volume $<15 \mathrm{ml}$ (testicular volume significantly correlates with testicular function; in particular, sperm parameters are subnormal in patients with a total testicular volume (right plus left testicular volume) below $30 \mathrm{ml}$ as analysed by orchidometry) ${ }^{11}$ and past or present cryptorchidism or varicocele.

\section{Female partners}

Gynaecological evaluation had previously excluded patients with female partners showing ovulatory dysfunction, tubal obstruction, urogenital infections and endometriosis. In addition, subjects' partners showed normal ultrasound findings of the uterus and ovaries and normal ovarian reserve.

\section{Measurement of glycosylated haemoglobin}

Levels of HbA1c were determined by high-pressure liquid chromatography. HPLC equipment (Variant II model), as well as calibration and control standards, was from Bio-Rad (Hercules, CA, USA). Reference ranges were $3.7 \%-6.2 \%$.

\section{Measurement of serum hormone concentrations}

The hormone assays were performed by electrochemiluminescence with a Hitachi-Roche device (Cobas 6000; Roche Diagnostics, Indianapolis, IN, USA). The reference intervals were as follows: $\mathrm{TSH}=0.3-4.2 \mathrm{mUI} \mathrm{ml} \mathrm{m}^{-1}, \mathrm{LH}=1.6-9.0 \mathrm{mUI} \mathrm{ml}^{-1}, \mathrm{FSH}=2.0$ $12.0 \mathrm{mUI} \mathrm{m}{ }^{-1}$, $17 \beta$-estradiol $=8.0-43.0 \mathrm{pg} \mathrm{ml}^{-1}$, total testosterone $=2.8-8.0 \mathrm{ng} \mathrm{ml}^{-1}$, prolactin $=4.0-15.0 \mathrm{ng} \mathrm{ml}^{-1}$, thyroglobulin antibody $\leqslant 100.0 \mathrm{mUI} \mathrm{ml}^{-1}$ and thyroid peroxidase antibody $=0.0$ $34.0 \mathrm{mUI} \mathrm{ml}{ }^{-1}$.

\section{Ultrasound evaluation}

All patients underwent prostate-vesicular transrectal ultrasonography before and $1 \mathrm{~h}$ after ejaculation following 1 day of sexual abstinence

Table 1 Serum hormone concentrations of patients and controls

\begin{tabular}{|c|c|c|c|c|c|c|c|c|}
\hline Groups & $\begin{array}{l}\text { TSH } \\
\left(m U / m I^{-1}\right)\end{array}$ & $\begin{array}{l}F S H \\
\left(m U I m I^{-1}\right)\end{array}$ & $\begin{array}{l}L H \\
\left(m U I m I^{-1}\right)\end{array}$ & 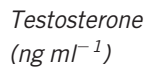 & 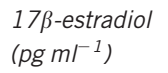 & $\begin{array}{l}\text { Prolactin } \\
\left(\mathrm{ng} \mathrm{ml^{-1 }}\right)\end{array}$ & $\begin{array}{l}A b T g \\
\left(U / \mathrm{m} I^{-1}\right)\end{array}$ & $\begin{array}{l}\text { AbTPO } \\
\left(U I \mathrm{ml}^{-1}\right)\end{array}$ \\
\hline $\begin{array}{l}\text { A: Patients with DM } \\
(\mathrm{HbA1 \textrm {C }}<7 \%)\end{array}$ & $1.4 \pm 2.0$ & $4.2 \pm 5.0$ & $3.2 \pm 3.0$ & $5.6 \pm 3.0$ & $13.0 \pm 10.0$ & $6.0 \pm 4.0$ & $33.0 \pm 20.0$ & $12.0 \pm 8.0$ \\
\hline $\begin{array}{l}\text { C: Patients with DM } \\
(\mathrm{HbA} 1 \mathrm{c}>10 \%)\end{array}$ & $1.2 \pm 2.0$ & $3.9 \pm 3.0$ & $2.9 \pm 4.0$ & $5.4 \pm 3.0$ & $11.0 \pm 10.0$ & $4.0 \pm 5.0$ & $23.0 \pm 10.0$ & $15.0 \pm 4.0$ \\
\hline
\end{tabular}

Abbreviations: AbTg, thyreoglobulin antibodies; AbTPO, thyreoperoxidase antibodies; DM, diabetes mellitus; FSH, follicle-stimulating hormone; HbA1c, glycosylated haemoglobin; LH, luteinzing hormone; OAT, oligoasthenoteratozoospermia; TSH, thyroid-stimulating hormone. 
using a transrectal 7.5 MHz biplan biconvex transducer (Esaote GPX Megas, Genova, Italy). We arbitrarily chose to examine patients after only 1 day of sexual abstinence to minimize factors that promote stasis in the SV.

The following SV ultrasound parameters were recorded for each patient by the same blind operator (SLV): (i) body APD; (ii) fundus APD; (iii) parietal thicknesses of the right and left SVs; and (iv) numbers of polycyclic areas within both SVs. These findings were used to calculate the following parameters: (i) F/B ratio; (ii) difference in parietal thickness between the right and the left SV; and (iii) preand post-ejaculatory APD difference. The measurements were repeated once and the results were expressed as the mean in the final report. For a measurement below $1 \mathrm{~mm}$, the calculations were performed on ultrasound paper with a millimetric measurement system.

The protocol was approved by the Institutional Review Board, and informed written consent was obtained from each patient and control subject.

\section{Statistical analysis}

Results are reported as mean \pm s.e.m. throughout the study. The data were analysed by one-way analysis of variance (ANOVA) followed by the Duncan's multiple range test and a unpaired $t$-test for direct comparisons between two groups. Statistical analysis was performed using SPSS 9.0 for Windows (SPSS Inc., Chicago, IL, USA). A $P$ value less than 0.05 was accepted as statistically significant.

\section{RESULTS}

There were no statistically significant differences among patients with DM with regard to age and BMI (low dispersion, more than 50\% of patients were between the first and third quartiles).

Among the different groups we considered, ANOVA did not reveal any significant difference in testosterone levels (Table 1). Hence, we did not include this covariate, which could affect SV parameters, in the statistical analysis. In addition, there were no significant differences among the groups as far as testicular size, prostate volume, and epididymal head and tail sizes by scrotal ultrasound scan (Table 2).

All patients with DM had a significantly higher body APD, fundus APD and F/B ratio compared with controls $(P<0.05$; $t$-test). Among the patient groups, Group $C$ had a significantly higher SV body and fundus APD $(P<0.05 v s$. Groups A and B; one-way ANOVA followed by Duncan test). In addition, Group $\mathrm{C}$ had a significantly higher F/B ratio compared to the other diabetic groups $(P<0.05 v s$. Group $A$ and Group B; one-way ANOVA followed by Duncan test) (Table 3).

No significant differences were found between patients with DM and controls in parietal thickness, difference in parietal thickness between the right and left SV, or the number of polycyclic areas (one-way ANOVA followed by Duncan test) (Table 3).

Patients with DM had a significantly lower $(P<0.05$; $t$-test $)$ pre- and post-ejaculatory difference in body SV APD compared with controls. Groups A $(0.40 \pm 0.06 \mathrm{~mm})$ and B $(0.46 \pm 0.16 \mathrm{~mm})$ had a similar pre- and post-ejaculatory difference of the body SV APD, whereas this difference was significantly lower in Group C $(0.10 \pm 0.06 \mathrm{~mm})$ $(P<0.05$ vs. Groups A and B; one-way ANOVA followed by Duncan test) (Table 3 ).

\section{Semen analysis}

Patients with DM had seminal leukocyte concentrations significantly higher compared with healthy and diseased control groups $(P<0.05)$. In addition, patients of Group $C$ had a higher concentration leukocytes compared with the other DM groups $(P<0.05)$. In addition, Group C showed significantly lower values for all conventional sperm parameters (concentration, progressive motility and percentage of normal forms), compared with the other groups of patients with DM and control groups $(P<0.05)$ (Table 4). Finally, ejaculate volume did not differ significantly between patients with DM and controls or among the groups of patients with DM.

\section{DISCUSSION}

This study confirmed that the SVs of infertile patients with type $2 \mathrm{DM}$ and DN had particular characteristics on ultrasound. In addition, these characteristics were accentuated in patients with worse glycaemic control. Finally, the patients with DM also showed a lower sperm quality compared with controls; in particular, they had higher seminal leukocyte concentrations, but a similar ejaculate volume.

Neuropathy, a frequent complication of DM, can cause not only sexual dysfunction but also infertility by altering SV function. ${ }^{12} \mathrm{SV}$ dysfunction is one of the factors thought to contribute to male infertility. The following mechanisms have been proposed:

1. SV dysfunction may relay to inflammation. It is well known that MAGI have a negative impact on sperm parameters ${ }^{13}$ and cause a leukocyte-related reactive oxygen species overproduction. Both events are directly related to extension of the inflammatory process. Indeed, prostate vesiculitis is associated with more severe sperm production impairment and higher reactive oxygen species production than prostatitis alone.

2. The secretory capacity of SV, evaluated by various biochemical markers (e.g., fructose), has been used to assess their function and/or to localize an obstruction. Recently, seminal fructose levels have been proposed as marker of sperm motility. Indeed, studies have shown that both corrected fructose (log (sperm concentration) multiplied by seminal fructose) and true corrected fructose (log (motile sperm concentration) multiplied by seminal fructose) are mathematical measures correlating with sperm motility in cases of asthenozoospermia. ${ }^{14}$

3. Finally, a recent $\operatorname{study}^{15}$ in patients with MAGI found an association between hyperechoic SV, dilated ejaculatory ducts or duct calcifications, and higher levels of seminal plasma interleukin-8, a reliable marker of prostatic inflammatory diseases and possibly a marker of male genital tract inflammation.

Table 2 Scrotal and prostate ultrasound parameters of patients and controls

\begin{tabular}{|c|c|c|c|c|}
\hline Groups & Prostate volume $\left(\mathrm{cm}^{3}\right)$ & Testicular volume (ml) & Epididymal head size ( $\mathrm{mm}$ ) & Epididymal tail size $(\mathrm{mm})$ \\
\hline A: Patients with DM ( $\mathrm{HbA} 1 \mathrm{c}<7 \%)$ & $32.0 \pm 6.0$ & $19.0 \pm 3.0$ & $10.0 \pm 2.0$ & $5.0 \pm 1.0$ \\
\hline B: Patients with DM (HbA1c $7 \%-10 \%)$ & $34.0 \pm 2.0$ & $21.0 \pm 2.0$ & $9.0 \pm 3.0$ & $4.0 \pm 2.0$ \\
\hline C: Patients with DM (HbA1c >10\%) & $33.5 \pm 4.0$ & $20.0 \pm 2.0$ & $10.0 \pm 1.0$ & $4.0 \pm 1.0$ \\
\hline OAT control group & $34.0 \pm 2.0$ & $22.0 \pm 4.0$ & $9.0 \pm 3.0$ & $5.0 \pm 1.0$ \\
\hline Fertile control group & $35.6 \pm 8.0$ & $21.0 \pm 5.0$ & $10.0 \pm 2.0$ & $4.0 \pm 2.0$ \\
\hline
\end{tabular}

Abbreviations: DM, diabetes mellitus; HbA1c, glycosylated haemoglobin; OAT, oligoasthenoteratozoospermia. 
Table 3 Ultrasound evaluation of seminal vesicles of patients and controls

\begin{tabular}{|c|c|c|c|c|c|c|c|}
\hline Groups & $\begin{array}{l}\text { Body APD } \\
(\mathrm{mm})\end{array}$ & $\begin{array}{l}\text { Fundus APD } \\
(\mathrm{mm})\end{array}$ & F/B ratio & $\begin{array}{l}\text { Parietal thickness } \\
(\mathrm{mm})\end{array}$ & $\begin{array}{l}\text { Parietal differences } \\
(\mathrm{mm})\end{array}$ & $\begin{array}{l}\text { No. of polycyclical } \\
\text { areas }\end{array}$ & $\begin{array}{l}\text { Pre- and post-ejaculatory } \\
\text { APD difference }(\mathrm{mm})\end{array}$ \\
\hline $\begin{array}{l}\text { A: Patients with DM } \\
(\mathrm{HbAlc}<7 \%)\end{array}$ & $12.0 \pm 2.0^{*}$ & $40.0 \pm 10.0^{*}$ & $2.6 \pm 3.0^{*}$ & $0.82 \pm 0.30$ & $0.82 \pm 0.20$ & $1.0 \pm 1.0$ & $0.40 \pm 0.06^{*}$ \\
\hline $\begin{array}{r}\text { B: Patients with DM } \\
\text { (HbAlc 7\%-10\%) }\end{array}$ & $12.0 \pm 3.0^{*}$ & $40.0 \pm 8.0^{*}$ & $2.6 \pm 2.0^{*}$ & $0.84 \pm 0.20$ & $0.81 \pm 0.30$ & $2.0 \pm 2.0$ & $0.46 \pm 0.16^{*}$ \\
\hline $\begin{array}{l}\text { C: Patients with DM } \\
(\text { HbAlc }>10 \%)\end{array}$ & $17.0 \pm 4.0^{* \dagger}$ & $50.0 \pm 10.0^{* *}$ & $3.6 \pm 2.0^{* \dagger}$ & $0.81 \pm 0.30$ & $0.83 \pm 0.40$ & $1.0 \pm 1.0$ & $0.10 \pm 0.06^{* \dagger}$ \\
\hline OAT control group & $8.0 \pm 2.0$ & $12.0 \pm 2.0$ & $1.0 \pm 0.6$ & $0.80 \pm 0.40$ & $0.81 \pm 0.20$ & $2.0 \pm 1.0$ & $1.20 \pm 0.60$ \\
\hline Fertile control group & $8.0 \pm 3.0$ & $12.0 \pm 4.0$ & $1.0 \pm 0.4$ & $0.80 \pm 0.30$ & $0.82 \pm 0.30$ & $2.0 \pm 2.0$ & $1.28 \pm 0.80$ \\
\hline
\end{tabular}

Abbreviations: APD, antero-posterior diameter; F/B, fundus/body ratio.

$* P<0.05$ vs. OAT and fertile controls.

${ }^{\dagger} P<0.05$ vs. DM patients with $\mathrm{HbA} 1 \mathrm{c}<7 \%, \mathrm{HbA} 1 \mathrm{c} 7 \%-10 \%$ and controls (OAT and fertile).

SV dysfunction is one possible cause of sexual impairment. A previous study showed that urogenital infections may affect sexual function by causing premature ejaculation and erectile dysfunction. ${ }^{16}$ In particular, more data link prostatitis to premature ejaculation than prostatitis to erectile disorders, and there is even more evidence which links lower urinary tract symptoms and sexual dysfunction. However, there is no direct evidence regarding the influence of SV dysfunction on ejaculation and erection. ${ }^{16}$ Nevertheless, some studies have indicated that prostate inflammation often involves both SV, ${ }^{13,14}$ and this could have multiple effects on sexual function.

There are no reported histopathological characteristics of SVs in patients with DM. The only data come from animal studies describing a reduction in the weight of male accessory sex glands that was counteracted by insulin treatment. ${ }^{17}$ Another recent study indicated that Solanum lycocarpum St. Hill, a native shrub common in the Brazilian savannah employed in folk medicine to manage diabetes, increased SV weights in male rats. ${ }^{18}$ In addition, previous reports have shown significant changes in the sympathetic innervation of the SV during streptozotocin-induced DM, and alterations in the reuptake, release and synthesis of the neurotransmitter noradrenaline have been implicated in changes in the tissue concentration of this amine. It is possible that the observed changes are related to the remodelling and regrowth of sympathetic nerve endings damaged in the early stages of hyperglycemia. These changes may also contribute to ejaculation disorders in patients with DM. ${ }^{19}$ Another study showed a decrease in the sensitivity of the VIP receptor/effector system in SV membranes from streptozotocin-treated rats, suggesting a physiopathological role for VIP in the seminal neuropathy observed in DM patients. ${ }^{20}$

Ultrasonographic examinations are useful for the diagnosis of diabetic neuropathy. A recent study evaluated the cross-sectional area and echo intensity of the peripheral nerve at the carpal tunnel, the median nerve proximal to the wrist and the tibial nerve at the ankle. The data revealed a significant increase in nerve cross-sectional area and hypoechoic area in patients with DM compared with controls. ${ }^{21}$ Cholecystomegaly was found in patients with type 2 diabetics to be significantly correlated with age, BMI and the severity of autonomic neuropathy. In male type $2 \mathrm{DM}$ patients, gallbladder volume correlated significantly with low-density lipoprotein cholesterol levels. In female type $2 \mathrm{DM}$ patients, gallbladder volume correlated significantly with waist/hip ratio. Gallbladder volume also showed a significant correlation with proliferative diabetic retinopathy but not with glycaemic control, microalbuminuria, hypertension or the duration of $\mathrm{DM}^{22}$ In addition, ultrasonographic measurement of gastric emptying time successfully and non-invasively identifies abnormal gastric motility in different types of disease. ${ }^{23}$ Gastroparesis is a complication of DM and can be documented using an ultrasonographic method. Poor glucose control and autonomic neuropathy are associated with gastroparesis. $^{24}$

\section{CONCLUSIONS}

To our knowledge, this is the first study exploring the ultrasound characteristics of the SV in relation to glycaemic control in infertile

\section{Table 4 Sperm analysis of patients and controls}

\begin{tabular}{|c|c|c|c|c|c|c|}
\hline Groups & $\begin{array}{l}\text { Sperm concentration } \\
\left(10^{6} \mathrm{ml}^{-1}\right)\end{array}$ & $\begin{array}{l}\text { Total sperm no. } \\
\left(10^{6} \text { ejaculated }\right)\end{array}$ & $\begin{array}{l}\text { Progressive motility } \\
(\%)\end{array}$ & $\begin{array}{l}\text { Morphology } \\
\text { (\% normal forms) }\end{array}$ & $\begin{array}{l}\text { Leukocytes } \\
\left(10^{6} \mathrm{ml}^{-1}\right)\end{array}$ & $\begin{array}{l}\text { Ejaculate volume } \\
(\mathrm{ml})\end{array}$ \\
\hline $\begin{array}{l}\text { A: Patients with DM } \\
\quad(\mathrm{HbA} 1 \mathrm{c}<7 \%)\end{array}$ & $52.0(36.0-84.0)$ & $131.0(90.5-186.0)$ & $42.0(35.0-50.0)$ & $16.0(8.0-31.0)^{\mathrm{b}}$ & $1.3(0.6-2.2)^{a}$ & $3.2(1.2-4.9)$ \\
\hline $\begin{array}{l}\text { B: Patients with DM } \\
\text { (HbA1c } 7 \%-10 \%)\end{array}$ & $30.0(22.0-72.0)^{b, c}$ & $83.0(40.0-110.0)^{b, c}$ & $32.0(22.0-42.0)^{b, c}$ & $10.0(6.0-30.0)^{\mathrm{b}, \mathrm{c}}$ & $1.5(0.7-2.4)^{a}$ & $3.1(1.1-4.8)$ \\
\hline $\begin{array}{l}\text { C: Patients with DM } \\
\quad(\mathrm{HbA} 1 \mathrm{c}>10 \%)\end{array}$ & $16.0(10.0-40.0)^{b, c, d}$ & $44.0(21.0-66.0)^{\mathrm{b}, \mathrm{c}, \mathrm{d}}$ & $20.0(14.0-36.0)^{\mathrm{b}, \mathrm{c}, \mathrm{d}}$ & $7.0(2.0-18.0)^{\mathrm{b}, \mathrm{c}, \mathrm{d}}$ & $2.6(0.9-3.6)^{a, c}$ & $3.0(1.3-5.0)$ \\
\hline OAT control group & $7.5(2.0-13.0)^{\mathrm{b}}$ & $17.0(12.0-20)^{b}$ & $15.8(10.0-24.0)^{b}$ & $4.0(1.0-8.0)^{b}$ & $0.8(0.1-1.9)$ & $3.2(1.0-5.2)$ \\
\hline Fertile control group & 57.5 (40.0-90.0) & $144.0(100.0-212.0)$ & $48.8(41.0-56.0)$ & $38.0(30.0-44.0)$ & $0.4(0.1-1.1)$ & $2.9(1.3-5.4)$ \\
\hline
\end{tabular}

Values are medians, and the $10^{\text {th }}-90^{\text {th }}$ percentiles are shown in parentheses.

Abbreviations: $\mathrm{HbA1c}$, glycosylated haemoglobin; OAT, oligoasthenoteratozoospermia.

${ }^{a} P<0.05$ vs. OAT and fertile control groups.

${ }^{\mathrm{b}} P<0.05$ vs. fertile control group.

${ }^{c} P<0.05$ vs. patients with diabetes mellitus ( $\mathrm{HbA} 1 \mathrm{c}<7 \%$ ).

${ }^{d} P<0.05$ vs. patients with diabetes mellitus ( $\mathrm{HbA} 1 \mathrm{c} 7 \%-10 \%$ ). 
patients with DM and DN and without other known causes of infertility. The lack of data on SV function in patients with DM is of particular relevance given the increasing interest of the negative impact of DM on sperm parameters and consequently on male reproductive function. ${ }^{25}$ Future studies are needed to clarify the association between these ultrasound signs and the increased seminal leukocytes concentration in these patients.

\section{AUTHOR CONTRIBUTIONS}

SLV, RAC, RDA, EV and AEC carried out the study, analysed the data and wrote the manuscript. SLV and AEC were involved in the study design, data management and analysis of the study.

\section{COMPETING FINANCIAL INTERESTS}

The authors declare no conflict of interest.

\section{ACKNOWLEDGMENTS}

We are grateful to Dr Flavia Di Bari and Dr Michele Salemi for their technical assistance in statistical analysis.

1 La Vignera S, Vicari E, Condorelli R, D'Agata R, Calogero AE. Ultrasound characterization of the seminal vesicles in infertile patients with type 2 diabetes mellitus. Eur J Radiol; e-pub ahead of print 25 August 2010; doi:10.1016/ j.ejrad.2010.08.001.

2 La Vignera S. Seminal vesicles and diabetic neuropathy: ultrasound evaluation J Androl; e-pub ahead of print 16 December 2010; doi:10.2164/jandrol.110.011676.

3 Vicari E, Calogero AE, Valenti D, Condorelli R, La Vignera S. Relationship between degree of neuropathy and post-ejaculatory seminal vesicles voiding and seminal leukocyte concentrations in diabetic patients. J Endocrinol Invest 2008; 31 (Suppl): 17

4 Dyck PJ. Detection, characterization, and staging of polyneuropathy: assessed in diabetics. Muscle Nerve 1988; 11: 21-32.

5 Vinik Al, Maser RE, Mitchell BD, Freeman R. Diabetic autonomic neuropathy. Diabetes Care 2003; 26: 1553-79.

6 World Health Organization. WHO Laboratory Manual for the Examination of Human Semen and Sperm-Cervical Mucus Interaction. 4th ed. Cambridge: Cambridge University Press; 1999.

7 Dandona P, Rosenberg MT. A pratical guide to male hypogonadism in the primary care setting. Int J Clin Pract 2010; 64: 682-96.

8 Walsh JP, Pullan PT. Hyperprolactinemia in males: a heterogeneous disorder. Aust NZ J Med 1997; 27: 385-90.
9 Cooper DS. Subclinical hypothyroidism. N Eng/ J Med 2001; 345: 260-5.

10 Corona G, Jannini EA, Lotti F, Boddi V, de Vita G et al. Premature and delayed ejaculation: two ends of a single continuum influenced by hormonal milieu. Int $J$ Androl 2011; 34: 41-8.

11 Sakamoto H, Ogawa Y, Yoshida H. Relationship between testicular volume and testicular function: comparison of the Prader orchidometric and ultrasonographic measurement in patients with infertility. Asian J Androl 2008; 10: 319-24.

12 Ali ST, Shaikh RN, Siddiqi NA, Siddiqi PQ. Semen analysis in insulin-dependent/noninsulin-dependent diabetic men with/without neuropathy. Arch Androl 1993; 30: 4754.

13 Vicari E. Seminal leukocyte concentration and related specific reactive oxygen species production in patients with male accessory gland infections. Hum Reprod 1999; 14: 2025-30.

14 Vicari E, La Vignera S, Castiglione R, Calogero AE. Sperm parameter abnormalities, low seminal fructose and reactive oxygen species overproduction do not discriminate patients with unilateral or bilateral post-infectious inflammatory prostato-vesiculoepididymitis. J Endocrinol Invest 2006; 29: 18-25.

15 Lotti F, Corona G, Mancini M, Filimberti E, Degli Innocenti S et al. Ultrasonographic and clinical correlates of seminal plasma interleukin-8 levels in patients attending an andrology clinic for infertility. Int J Androl; e-pub ahead of print 24 October 2010; doi:10.1111/j.1365-2605.2010.01121.x.

16 Schultheiss D. Urogenital infections and male sexuality: effects on ejaculation and erection. Andrologia 2008; 40: 125-9.

17 Jackson FL, Hutson JC. Altered responses to androgen in diabetic male rats. Diabetes 1984; 33: 819-24.

18 Soares Mota MR, Schwarz A, Bernardi MM, Maiorka PC, Spinosa Hde S. Toxicological evaluation of $10 \%$ Solanum lycocarpum St. Hill fruit consumption in the diet of growing rats: hematological, biochemical and histopathological effects. Exp Toxicol Pathol 2010; 62: 549-53.

19 Morrison JF, Dhanasekaran S, Sheen R, Frampton CM, Mensah-Brown E. The effect of streptozotocin-induced diabetes on the rat seminal vesicle: a possible pathophysiological basis for disorders of ejaculation. Ann NY Acad Sci 2006; 1084: 267-79.

20 Rodriguez-Pena MS, Guijarro LG, Juarranz MG, Rodriguez-Henche N, Bajo AM et al. Analysis of vasoactive intestinal peptide receptors and the $G$ protein regulation of adenylyl cyclase in seminal vesicle membranes from streptozotocin-diabetic rats. Cell Signal 1994; 6: 147-56.

21 Watanabe T, Ito H, Sekine A, Katano Y, Nishimura T et al. Sonographic evaluation of the peripheral nerve in diabetic patients: the relationship between nerve conduction studies, echo intensity, and cross-sectional area. J Ultrasound Med 2010; 29: 697708.

22 Agarwal AK, Miglani S, Singla S, Garg U, Dudeja RK et al. Ultrasonographic evaluation of gallbladder volume in diabetics. J Assoc Physicians India 2004; 52: 962-5.

23 Dorlars D, Schilling D, Riemann JF. The feasibility of ultrasonography for the evaluation of stomach motility disorders. Dtsch Med Wochenschr 1994; 119: 57580.

24 Moldovan C, Dumitrascu DL, Demian L, Brisc C, Vatca L et al. Gastroparesis in diabetes mellitus: an ultrasonographic study. Rom J Gastroenterol 2005; 14: 19-22.

25 Bener A, Al-Ansari AA, Zirie M, Al-Hamaq AO. Is male fertility associated with type 2 diabetes mellitus? Int Urol Nephrol 2009; 41: 777-84. 